\title{
Some inequalities for Cesàro means of double Vilenkin-Fourier series
}

T. Tepnadze ${ }^{1}$ and L.E. Persson ${ }^{1 *}$

"Correspondence: larserik6pers@gmail.com

The Artic University of Norway, Narvik, Norway

\begin{abstract}
In this paper, we state and prove some new inequalities related to the rate of $L^{p}$ approximation by Cesàro means of the quadratic partial sums of double Vilenkin-Fourier series of functions from $L^{p}$.

MSC: $42 \mathrm{C} 10 ; 42 \mathrm{~B} 25$

Keywords: Inequalities; Approximation; Vilenkin system; Vilenkin-Fourier series; Cesàro means; Convergence in norm
\end{abstract}

\section{Introduction}

Let $N_{+}$denote the set of positive integers, and let $N:=N_{+} \cup\{0\}$. Let $m:=\left(m_{0}, m_{1}, \ldots\right)$ be a sequence of positive integers not less than 2 . Denote by $Z_{m_{k}}:=\left\{0,1, \ldots, m_{k}-1\right\}$ the additive group of integers modulo $m_{k}$. Define the group $G_{m}$ as the complete direct product of the groups $Z_{m_{j}}$ with the product of the discrete topologies of $Z_{m_{j}}$.

The direct product of the measures

$$
\mu_{k}(\{j\}):=\frac{1}{m_{k}} \quad\left(j \in Z_{m_{k}}\right)
$$

is the Haar measure on $G_{m}$ with $\mu\left(G_{m}\right)=1$. If the sequence $m$ is bounded, then $G_{m}$ is called a bounded Vilenkin group. In this paper, we consider only bounded Vilenkin groups. The elements of $G_{m}$ can be represented by sequences $x:=\left(x_{0}, x_{1}, \ldots, x_{j}, \ldots\right)\left(x_{j} \in Z_{m_{j}}\right)$. The group operation + in $G_{m}$ is given by

$$
x+y=\left(\left(x_{0}+y_{0}\right) \bmod m_{0}, \ldots,\left(x_{k}+y_{k}\right) \bmod m_{k}, \ldots\right)
$$

for $x:=\left(x_{0}, \ldots, x_{k}, \ldots\right)$ and $y:=\left(y_{0}, \ldots, y_{k}, \ldots\right) \in G_{m}$. The inverse of + will be denoted by - .

It is easy to give a base for the neighborhoods of $G_{m}$ :

$$
\begin{aligned}
& I_{0}(x):=G_{m}, \\
& I_{n}(x):=\left\{y \in G_{m} \mid y_{0}=x_{0}, \ldots, y_{n-1}=x_{n-1}\right\}
\end{aligned}
$$

for $x \in G_{m}$ and $n \in N$. Define $I_{n}:=I_{n}(0)$ for $n \in N_{+}$. Set $e_{n}:=(0, \ldots, 0,1,0, \ldots) \in G_{m}$, where the $n$th coordinate of which is 1 , and the rest are zeros $(n \in N)$.

(c) The Author(s) 2018. This article is distributed under the terms of the Creative Commons Attribution 4.0 International License (http://creativecommons.org/licenses/by/4.0/), which permits unrestricted use, distribution, and reproduction in any medium, provided you give appropriate credit to the original author(s) and the source, provide a link to the Creative Commons license, and indicate if changes were made. 
We define the so-called generalized number system based on $m$ as follows: $M_{0}:=1$, $M_{k+1}:=m_{k} M_{k}(k \in N)$. Then every $n \in N$ can be uniquely expressed as $n=\sum_{j=0}^{\infty} n_{j} M_{j}$, where $n_{j} \in Z_{m_{j}}\left(j \in N_{+}\right)$, and only a finite number of $n_{j}$ differ from zero. We also use the following notation: $|n|:=\max \left\{k \in N: n_{k} \neq 0\right\}$ (i.e., $M_{|n|} \leq n<M_{|n|+1}, n \neq 0$ ). For $x \in G_{m}$, we denote $|x|:=\sum_{j=0}^{\infty} \frac{x_{j}}{M_{j+1}}\left(x_{j} \in Z_{m_{j}}\right)$.

Next, we introduce on $G_{m}$ an orthonormal system, which is called the Vilenkin system. First, we define the complex-valued functions $r_{k}(x): G_{m} \rightarrow C$, the generalized Rademacher functions, as follows:

$$
r_{k}(x):=\exp \frac{2 \pi i x_{k}}{m_{k}} \quad\left(i^{2}=-1, x \in G_{m}, k \in N\right) .
$$

Now we define the Vilenkin system $\psi:=\left(\psi_{n}: n \in N\right)$ on $G_{m}$ as

$$
\psi_{n}(x):=\prod_{k=0}^{\infty} r_{k}^{n_{k}}(x) \quad(n \in N)
$$

In particular, if $m=2$, then we call this system the Walsh-Paley system. Each $\psi_{n}$ is a character of $G_{m}$, and all characters of $G_{m}$ are of this norm. Moreover, $\psi_{n}(-x)=\bar{\psi}_{n}(x)$.

The Dirichlet kernels are defined by

$$
D_{n}:=\sum_{k=0}^{n-1} \psi_{k} \quad\left(n \in N_{+}\right) .
$$

Recall that (see [20] or [23])

$$
D_{M_{n}}(x)= \begin{cases}M_{n} & \text { if } x \in I_{n} \\ 0 & \text { if } x \notin I_{n}\end{cases}
$$

The Vilenkin system is orthonormal and complete in $L^{1}\left(G_{m}\right)$ (see [1]).

Next, we introduce some notation from the theory of two-dimensional Vilenkin system. Let $\tilde{m}$ be a sequence like $m$. The relation between the sequences $\left(\tilde{m}_{n}\right)$ and $\left(\tilde{M}_{n}\right)$ is the same as between sequences $\left(m_{n}\right)$ and $\left(M_{n}\right)$. The group $G_{m} \times G_{\tilde{m}}$ is called a two-dimensional Vilenkin group. The normalized Haar measure is denoted by $\mu$ as in the one-dimensional case. We also suppose that $m=\tilde{m}$ and $G_{m} \times G_{\tilde{m}}=G_{m}^{2}$.

The norm of the space $L^{p}\left(G_{m}^{2}\right)$ is defined by

$$
\|f\|_{p}:=\left(\int_{G_{m}^{2}}|f(x, y)|^{p} d \mu(x, y)\right)^{1 / p} \quad(1 \leq p<\infty) .
$$

Denote by $C\left(G_{m}^{2}\right)$ the class of continuous functions on the group $G_{m}^{2}$ endowed with the supremum norm.

For brevity in notation, we write $L^{\infty}\left(G_{m}^{2}\right)$ instead of $C\left(G_{m}^{2}\right)$.

The two-dimensional Fourier coefficients, the rectangular partial sums of the Fourier series, and the Dirichlet kernels with respect to the two-dimensional Vilenkin system are 
defined as follows:

$$
\begin{aligned}
& \widehat{f}\left(n_{1}, n_{2}\right):=\int_{G_{m}^{2}} f(x, y) \bar{\psi}_{n_{1}}(x) \bar{\psi}_{n_{2}}(y) d \mu(x, y), \\
& S_{n_{1}, n_{2}}(x, y, f):=\sum_{k_{1}=0}^{n_{1}-1} \sum_{k_{2}=0}^{n_{2}-1} \widehat{f}\left(k_{1}, k_{2}\right) \psi_{k_{1}}(x) \psi_{k_{2}}(y), \\
& D_{n_{1}, n_{2}}(x, y):=D_{n_{1}}(x) D_{n_{2}}(y),
\end{aligned}
$$

Denote

$$
\begin{aligned}
S_{n}^{(1)}(x, y, f) & :=\sum_{l=0}^{n-1} \widehat{f}(l, y) \bar{\psi}_{l}(x), \\
S_{m}^{(2)}(x, y, f) & :=\sum_{r=0}^{m-1} \widehat{f}(x, r) \bar{\psi}_{r}(y),
\end{aligned}
$$

where

$$
\widehat{f}(l, y)=\int_{G_{m}} f(x, y) \psi_{l}(x) d \mu(x)
$$

and

$$
\widehat{f}(x, r)=\int_{G_{m}} f(x, y) \psi_{r}(y) d \mu(y)
$$

The $(C,-\alpha)$ means of the double Vilenkin-Fourier series are defined as follows:

$$
\sigma_{n}^{-\alpha}(f, x, y)=\frac{1}{A_{n-1}^{-\alpha}} \sum_{j=1}^{n} A_{n-j}^{-\alpha-1} S_{j, j}(f, x, y),
$$

where

$$
A_{0}^{\alpha}=1, \quad A_{n}^{\alpha}=\frac{(\alpha+1) \cdots(\alpha+n)}{n !} .
$$

It is well known that (see [28])

$$
\begin{aligned}
& A_{n}^{\alpha}=\sum_{k=0}^{n} A_{k}^{\alpha-1}, \\
& A_{n}^{\alpha}-A_{n-1}^{\alpha}=A_{n}^{\alpha-1},
\end{aligned}
$$

and

$$
c_{1}(\alpha) n^{\alpha} \leq A_{n}^{\alpha} \leq c_{2}(\alpha) n^{\alpha},
$$

where positive constants $c_{1}$ and $c_{2}$ depend on $\alpha$. 
The dyadic partial moduli of continuity of a function $f \in L^{p}\left(G_{m}^{2}\right)$ in the $L^{p}$-norm are defined by

$$
\omega_{1}\left(f, \frac{1}{M_{n}}\right)_{p}=\sup _{u \in I_{n}}\|f(\cdot+u, \cdot)-f(\cdot, \cdot)\|_{p}
$$

and

$$
\omega_{2}\left(f, \frac{1}{M_{n}}\right)_{p}=\sup _{v \in I_{n}}\|f(\cdot, \cdot+v)-f(\cdot, \cdot)\|_{p}
$$

whereas the dyadic mixed modulus of continuity is defined as follows:

$$
\begin{aligned}
& \omega_{1,2}\left(f, \frac{1}{M_{n}}, \frac{1}{M_{m}}\right)_{p} \\
& =\sup _{(u, v) \in I_{n} \times I_{m}}\|f(\cdot+u, \cdot+v)-f(\cdot+u, \cdot)-f(\cdot, \cdot+v)+f(\cdot, \cdot)\|_{p} .
\end{aligned}
$$

It is clear that

$$
\omega_{1,2}\left(f, \frac{1}{M_{n}}, \frac{1}{M_{m}}\right)_{p} \leq \omega_{1}\left(f, \frac{1}{M_{n}}\right)_{p}+\omega_{2}\left(f, \frac{1}{M_{m}}\right)_{p} .
$$

The dyadic total modulus of continuity is defined by

$$
\omega\left(f, \frac{1}{M_{n}}\right)_{p}=\sup _{(u, v) \in I_{n} \times I_{n}}\|f(\cdot+u, \cdot+v)-f(\cdot, \cdot)\|_{p} .
$$

The problems of summability of partial sums and Cesàro means for Walsh-Fourier series were studied in $[2,13-19,21,22,25,26]$.

The convergence issue of Fejér (and Cesàro) means on the Walsh and Vilenkin groups for unbounded case were studied in [3-11].

In his monograph [27], Zhizhinashvili investigated the behavior of Cesàro $(C, \alpha)$-means for double trigonometric Fourier series in detail. Goginava [18] studied the analogous question in the case of the Walsh system. In particular, the following theorems were proved.

Theorem A Let $f$ belong to $L^{p}\left(G_{2}\right)$ for some $p \in[1, \infty]$ and $\alpha \in(0,1)$. Then, for any $2^{k} \leq$ $n<2^{k+1}(k, n \in N)$, we have the inequality

$$
\begin{aligned}
\left\|\sigma_{2^{k}}^{-\alpha}(f)-f\right\|_{p} \leq & c(\alpha)\left\{2^{k \alpha} \omega_{1}\left(f, 1 / 2^{k-1}\right)_{p}+2^{k \alpha} \omega_{2}\left(f, 1 / 2^{k-1}\right)_{p}\right. \\
& \left.+\sum_{r=0}^{k-2} 2^{r-k} \omega_{1}\left(f, 1 / 2^{r}\right)_{p}+\sum_{s=0}^{k-2} 2^{s-k} \omega_{2}\left(f, 1 / 2^{s}\right)_{p}\right\} .
\end{aligned}
$$


Theorem B Let $f$ belong to $L^{p}\left(G_{2}\right)$ for some $p \in[1, \infty]$ and $\alpha \in(0,1)$. Then, for any $2^{k} \leq$ $n<2^{k+1}(k, n \in N)$, we have the inequality

$$
\begin{aligned}
\left\|\sigma_{n}^{-\alpha}(f)-f\right\|_{p} \leq & c(\alpha)\left\{2^{k \alpha} k \omega_{1}\left(f, 1 / 2^{k-1}\right)_{p}+2^{k \alpha} k \omega_{2}\left(f, 1 / 2^{k-1}\right)_{p}\right. \\
& \left.+\sum_{r=0}^{k-2} 2^{r-k} \omega_{1}\left(f, 1 / 2^{r}\right)_{p}+\sum_{s=0}^{k-2} 2^{s-k} \omega_{2}\left(f, 1 / 2^{s}\right)_{p}\right\} .
\end{aligned}
$$

In this paper, we state and prove analogous results in the case of double Vilenkin-Fourier series. Our main results are the following theorems.

Theorem 1 Let $f$ belong to $L^{p}\left(G_{m}^{2}\right)$ for some $p \in[1, \infty]$ and $\alpha \in(0,1)$. Then, for any $M_{k} \leq$ $n<M_{k+1}(k, n \in N)$, we have the inequality

$$
\begin{aligned}
\left\|\sigma_{M_{k}}^{-\alpha}(f)-f\right\|_{p} \leq & c(\alpha)\left(\omega_{1}\left(f, 1 / M_{k-1}\right)_{p} M_{k}^{\alpha}+\omega_{2}\left(f, 1 / M_{l-1}\right)_{p} M_{k}^{\alpha}\right. \\
& \left.+\sum_{r=0}^{k-2} \frac{M_{r}}{M_{k}} \omega_{1}\left(f, 1 / M_{r}\right)_{p}+\sum_{s=0}^{k-2} \frac{M_{s}}{M_{k}} \omega_{2}\left(f, 1 / M_{s}\right)_{p}\right) .
\end{aligned}
$$

Theorem 2 Let $f$ belong to $L^{p}\left(G_{m}^{2}\right)$ for some $p \in[1, \infty]$ and $\alpha \in(0,1)$. Then, for any $M_{k} \leq$ $n<M_{k+1}(k, n \in N)$, we have the inequality

$$
\begin{aligned}
& \left\|\sigma_{n}^{-\alpha}(f)-f\right\|_{p} \\
& \leq c(\alpha)\left(\omega_{1}\left(f, 1 / M_{k-1}\right)_{p} M_{k}^{\alpha} \log n+\omega_{2}\left(f, 1 / M_{l-1}\right)_{p} M_{k}^{\alpha} \log n\right. \\
& \left.\quad+\sum_{r=0}^{k-2} \frac{M_{r}}{M_{k}} \omega_{1}\left(f, 1 / M_{r}\right)_{p}+\sum_{s=0}^{k-2} \frac{M_{s}}{M_{k}} \omega_{2}\left(f, 1 / M_{s}\right)_{p}\right) .
\end{aligned}
$$

To make the proofs of these theorems clearer, we formulate some auxiliary lemmas in Sect. 2. Some of these lemmas are new and of independent interest. Detailed proofs can be found in Sect. 3 .

\section{Auxiliary lemmas}

To prove Theorems 1 and 2, we need the following three lemmas (see [1, 12], and [8], respectively)

Lemma 1 Let $\alpha_{1}, \alpha_{2}, \ldots, \alpha_{n}$ be real numbers. Then

$$
\frac{1}{n} \int_{G}\left|\sum_{k=1}^{n} \alpha_{k} D_{k}(x)\right| d \mu(x) \leq \frac{c}{\sqrt{n}}\left(\sum_{k=1}^{n} \alpha_{k}^{2}\right)^{1 / 2} .
$$

Lemma 2 Let $\alpha_{1}, \alpha_{2}, \ldots, \alpha_{n}$ be real numbers. Then

$$
\frac{1}{n} \int_{G_{m}^{2}}\left|\sum_{k=1}^{n} \alpha_{k} D_{k}(x) D_{k}(y)\right| d \mu(x, y) \leq \frac{c}{\sqrt{n}}\left(\sum_{k=1}^{n} \alpha_{k}^{2}\right)^{1 / 2} .
$$


Lemma 3 Let $0 \leq j<n_{s} M_{s}$ and $0 \leq n_{s}<m_{s}$. Then

$$
D_{n_{s} M_{s}-j}=D_{n_{s} M_{s}}-\psi_{n_{s} M_{s}-1} \bar{D}_{j}
$$

We also need the following new nemmas of independent interest.

Lemma 4 Let $f$ belong to $L^{p}\left(G_{m}^{2}\right)$ for some $p \in[1, \infty]$. Then, for every $\alpha \in(0,1)$, we have the inequality

$$
\begin{aligned}
I & :=\frac{1}{A_{n}^{-\alpha}}\left\|\int_{G_{m}^{2}} \sum_{i=1}^{M_{k-1}} A_{n-i}^{-\alpha-1} D_{i}(u) D_{i}(v)[f(\cdot-u, \cdot-)-f(\cdot, \cdot)] d \mu(u, v)\right\|_{p} \\
& \leq \sum_{r=0}^{k-2} \frac{M_{r}}{M_{k}} \omega_{1}\left(f, 1 / M_{r}\right)_{p}+\sum_{s=0}^{k-2} \frac{M_{s}}{M_{k}} \omega_{2}\left(f, 1 / M_{s}\right)_{p},
\end{aligned}
$$

where $M_{k} \leq n<M_{k+1}$.

Lemma 5 Let $\alpha \in(0,1)$ and $p=M_{k}, M_{k}+1, \ldots$ Then

$$
I I:=\int_{G_{m}^{2}}\left|\sum_{i=1}^{M_{k}} A_{p-i}^{-\alpha-1} D_{i}(u) D_{i}(v)\right| d \mu(u, v) \leq c(\alpha)<\infty, \quad k=1,2, \ldots
$$

Lemma 6 We have the inequality

$$
I I I:=\int_{G_{m}^{2}}\left|\sum_{i=1}^{n} A_{n-i}^{-\alpha-1} D_{i}(u) D_{i}(v)\right| d \mu(u, v) \leq c(\alpha) \log n
$$

\section{The detailed proofs}

Proof of Lemma 3 Applying Abel's transformation, from (2) we get

$$
\begin{aligned}
I \leq & \frac{1}{A_{n}^{-\alpha}}\left\|\int_{G_{m}^{2}} \sum_{i=1}^{M_{k-1}-1} A_{n-i}^{-\alpha-2} \sum_{l=1}^{i} D_{i}(u) D_{i}(v)[f(\cdot-u, \cdot-v)-f(\cdot, \cdot)] d \mu(u, v)\right\|_{p} \\
& +\frac{1}{A_{n}^{-\alpha}}\left\|\int_{G_{m}^{2}} A_{n-M_{k-1}}^{-\alpha-1} \sum_{i=1}^{M_{k-1}} D_{i}(u) D_{i}(v)[f(\cdot-u, \cdot-v)-f(\cdot, \cdot)] d \mu(u, v)\right\|_{p} \\
:= & I_{1}+I_{2},
\end{aligned}
$$

where the first and second terms on the right side of inequality (5) are denoted by $I_{1}$ and $I_{2}$, respectively. 
For $I_{2}$, we have the estimate

$$
\begin{aligned}
I_{2} \leq & \frac{1}{A_{n}^{-\alpha}} \| \int_{G_{m}^{2}} A_{n-M_{k-1}}^{-\alpha-1} \sum_{r=1}^{k-2} \sum_{i=M_{r}}^{M_{r+1}-1} D_{i}(u) D_{i}(v) \\
& \times[f(\cdot-u, \cdot-v)-f(\cdot, \cdot)] \|_{p} d \mu(u, v) \\
\leq & \frac{1}{A_{n}^{-\alpha}} \| \int_{G_{m}^{2}} A_{n-M_{k-1}}^{-\alpha-1} \sum_{r=1}^{k-2} \sum_{i=M_{r}}^{M_{r+1}-1} D_{i}(u) D_{i}(v) \\
& \times\left[f(\cdot-u, \cdot-v)-S_{M_{r}, M_{r}}(\cdot-u, \cdot-v, f)\right] d \mu(u, v) \|_{p} \\
& +\frac{1}{A_{n}^{-\alpha}} \| \int_{G_{m}^{2}} A_{n-M_{k-1}}^{-\alpha-1} \sum_{r=1}^{k-2} \sum_{i=M_{r}}^{M_{r+1}-1} D_{i}(u) D_{i}(v) \\
& \times\left[S_{M_{r}, M_{r}}(\cdot-u, \cdot-v, f)-S_{M_{r}, M_{r}}(\cdot, \cdot, f)\right] d \mu(u, v) \|_{p} \\
& +\frac{1}{A_{n}^{-\alpha}} \| \int_{G_{m}^{2}} A_{n-M_{k-1}}^{-\alpha-1} \sum_{r=1}^{k-2} \sum_{i=M_{r}}^{M_{r+1}-1} D_{i}(u) D_{i}(v) \\
& \times\left[S_{M_{r}, M_{r}}(\cdot, \cdot, f)-f(\cdot, \cdot)\right] d \mu(u, v) \|_{p}+I_{23}, \\
& \\
&
\end{aligned}
$$

where the first, second, and third terms on the right side of inequality (6) are denoted by $I_{21}, I_{22}$, and $I_{23}$, respectively.

It is evident that

$$
\begin{aligned}
& \int_{G_{m}^{2}} \sum_{i=M_{r}}^{M_{r+1}-1} D_{i}(u) D_{i}(v)\left[S_{M_{r}, M_{r}}(\cdot-u, \cdot-v, f)-S_{M_{r}, M_{r}}(\cdot, \cdot, f)\right] d \mu(u, v) \\
& =\sum_{i=M_{r}}^{M_{r+1}-1}\left(\int_{G_{m}^{2}} D_{i}(u) D_{i}(v) S_{M_{r}, M_{r}}(\cdot-u, \cdot-v, f) d \mu(u, v)-S_{M_{r}, M_{r}}(\cdot, \cdot, f)\right) \\
& =\sum_{i=M_{r}}^{M_{r+1}-1}\left(S_{i}\left(\cdot, \cdot, S_{M_{r}, M_{r}}(f)\right)-S_{M_{r}, M_{r}}(\cdot, \cdot, f)\right) \\
& =\sum_{i=M_{r}}^{M_{r+1}-1}\left(S_{M_{r}, M_{r}}(\cdot, \cdot, f)-S_{M_{r}, M_{r}}(\cdot, \cdot, f)\right)=0 .
\end{aligned}
$$

Hence

$$
I_{22}=0 \text {. }
$$


Moreover, by the generalized Minkowski inequality, Lemma 2, and by (1) and (4) we obtain

$$
\begin{aligned}
I_{21} \leq & \frac{1}{A_{n}^{-\alpha}}\left|A_{n-M_{k-1}}^{-\alpha-1}\right| \sum_{r=1}^{k-2} \int_{G_{m}^{2}}\left|\sum_{i=M_{r}}^{M_{r+1}-1} D_{i}(u) D_{i}(v)\right| \\
& \times\left\|f(\cdot-u, \cdot-v)-S_{M_{r}, M_{r}}(\cdot-u, \cdot-v, f)\right\|_{p} d \mu(u, v) \\
\leq & \frac{c(\alpha)}{M_{k}} \sum_{r=1}^{k-2}\left(\omega_{1}\left(f, 1 / M_{r}\right)_{p}+\omega_{2}\left(f, 1 / M_{r}\right)_{p}\right) \\
& \times \int_{G_{m}^{2}}\left|\sum_{i=M_{r}}^{M_{r+1}-1} D_{i}(x) D_{i}(y)\right| d \mu(u, v) \\
\leq & c(\alpha) \sum_{r=1}^{k-2} \frac{M_{r}}{M_{k}}\left(\omega_{1}\left(f, 1 / M_{r}\right)_{p}+\omega_{2}\left(f, 1 / M_{r}\right)_{p}\right) .
\end{aligned}
$$

The estimation of $I_{23}$ is analogous to that of $I_{21}$ :

$$
I_{23} \leq c(\alpha) \sum_{r=1}^{k-2} \frac{M_{r}}{M_{k}}\left(\omega_{1}\left(f, 1 / M_{r}\right)_{p}+\omega_{2}\left(f, 1 / M_{r}\right)_{p}\right) .
$$

Analogously, we can estimate $I_{1}$ as follows:

$$
\begin{aligned}
& I_{1} \leq \frac{1}{A_{n}^{-\alpha}} \sum_{r=1}^{k-2} \| \int_{G_{m}^{2}} \sum_{i=M_{r}}^{M_{r+1}-1} A_{n-i}^{-\alpha-2} \sum_{l=1}^{i} D_{l}(u) D_{l}(v) \\
& \times\left[f(\cdot-u, \cdot-v)-S_{M_{r}, M_{r}}(\cdot-u, \cdot-v, f)\right] d \mu(u, v) \|_{p} \\
& +\frac{1}{A_{n}^{-\alpha}} \sum_{r=1}^{k-2} \| \int_{G_{m}^{2}} \sum_{i=M_{r}}^{M_{r+1}-1} A_{n-i}^{-\alpha-2} \sum_{l=1}^{i} D_{l}(u) D_{l}(v) \\
& \times\left[S_{M_{r}, M_{r}}(\cdot-u, \cdot-v, f)-S_{M_{r}, M_{r}}(\cdot, \cdot, f)\right] \|_{p} d \mu(u, v) \\
& +\frac{1}{A_{n}^{-\alpha}} \sum_{r=1}^{k-2} \| \int_{G_{m}^{2}} \sum_{i=M_{r}}^{M_{r+1}-1} A_{n-i}^{-\alpha-2} \sum_{l=1}^{i} D_{l}(u) D_{l}(v) \\
& \times\left[S_{M_{r}, M_{r}}(\cdot, \cdot, f)-f(\cdot, \cdot)\right] d \mu(u, v) \|_{p} \\
& \leq \frac{1}{A_{n}^{-\alpha}} \sum_{r=1}^{k-2} \int_{G_{m}^{2}}\left|\sum_{i=M_{r}}^{M_{r+1}-1} A_{n-i}^{-\alpha-2} \sum_{l=1}^{i} D_{l}(u) D_{l}(v)\right| \\
& \times\left\|f(\cdot-u, \cdot-v)-S_{M_{r}, M_{r}}(\cdot-u, \cdot-v, f)\right\|_{p} d \mu(u, v)
\end{aligned}
$$




$$
\begin{aligned}
& \quad+\frac{1}{A_{n}^{-\alpha}} \sum_{r=1}^{k-2} \int_{G_{m}^{2}}\left|\sum_{i=M_{r}}^{M_{r+1}-1} A_{n-i}^{-\alpha-2} \sum_{l=1}^{i} D_{l}(u) D_{l}(v)\right| \\
& \times\left\|S_{M_{r}, M_{r}}(\cdot, \cdot, f)-f(\cdot, \cdot)\right\|_{p} d \mu(u, v) \\
& \leq c(\alpha) M_{k}^{\alpha} \sum_{r=1}^{k-2} \sum_{i=M_{r}}^{M_{r+1}-1}(n-i)^{-\alpha-2} i\left(\omega_{1}\left(f, 1 / M_{r}\right)_{p}+\omega_{2}\left(f, 1 / M_{r}\right)_{p}\right) \\
& \leq c(\alpha) M_{k}^{\alpha} \sum_{r=1}^{k-2} \sum_{i=M_{r}}^{M_{r+1}-1}\left(n-M_{r+1}-1\right)^{-\alpha-2} i\left(\omega_{1}\left(f, 1 / M_{r}\right)_{p}+\omega_{2}\left(f, 1 / M_{r}\right)_{p}\right) \\
& \leq c(\alpha) \sum_{r=0}^{k-2} \frac{M_{r}}{M_{k}}\left(\omega_{1}\left(f, 1 / M_{r}\right)_{p}+\omega_{2}\left(f, 1 / M_{r}\right)_{p}\right) .
\end{aligned}
$$

By combining (7)-(9) with (10) for $I$ we find that

$$
I \leq c(\alpha) \sum_{r=0}^{k-2} \frac{M_{r}}{M_{k}}\left(\omega_{1}\left(f, 1 / M_{r}\right)_{p}+\omega_{2}\left(f, 1 / M_{r}\right)_{p}\right) .
$$

The proof of Lemma 3 is complete.

Proof of Lemma 4 It is evident that

$$
\begin{aligned}
I I \leq & \int_{G_{m}^{2}}\left|\sum_{i=1}^{M_{k}-1} A_{p-M_{k}+i}^{-\alpha-1} D_{M_{k}-i}(u) D_{M_{k}-i}(v)\right| d \mu(u, v) \\
& +\left|A_{p-M_{k}}^{-\alpha-1}\right| \int_{G_{m}^{2}} D_{M_{k}}(u) D_{M_{k}}(v) d \mu(u, v) \\
:= & I I_{1}+I I_{2}
\end{aligned}
$$

where the first and second terms on the right side of inequality (12) are denoted by $I I_{1}$ and $I I_{2}$, respectively.

From (1) by $\left|A_{p-M_{k}}^{-\alpha-1}\right| \leq 1$ we get that

$$
I I_{2} \leq 1
$$

Moreover, by Lemma 3 we have that

$$
\begin{aligned}
I I_{1} \leq & \int_{G_{m}^{2}}\left|\sum_{i=1}^{M_{k}-1} A_{p-M_{k}+i}^{-\alpha-1} \bar{D}_{i}(u) \bar{D}_{i}(v)\right| d \mu(u, v) \\
& +\int_{G_{m}^{2}} D_{M_{k}}(u)\left|\sum_{i=1}^{M_{k}-1} A_{p-M_{k}+i}^{-\alpha-1} \bar{D}_{i}(v)\right| d \mu(u, v) \\
& +\int_{G_{m}^{2}} D_{M_{k}}(v)\left|\sum_{i=1}^{M_{k}-1} A_{p-M_{k}+i}^{-\alpha-1} \bar{D}_{i}(u)\right| d \mu(u, v)
\end{aligned}
$$




$$
\begin{aligned}
& \quad+\left|\sum_{i=1}^{M_{k}-1} A_{p-M_{k}+i}^{-\alpha-1}\right| \int_{G_{m}^{2}} D_{M_{k}}(u) D_{M_{k}}(v) d \mu(u, v) \\
& :=I I_{11}+I I_{12}+I I_{13}+I I_{14},
\end{aligned}
$$

where the first, second, third, and fourth terms on the right side of inequality (14) are denoted by $I I_{11}, I I_{12}, I I_{13}$, and $I I_{14}$ respectively.

From (1) and (4) it follows that

$$
I I_{14} \leq c(\alpha) \sum_{v=1}^{\infty} v^{-\alpha-1}<\infty
$$

By Applying Abel's transformation, in view of Lemma 2, we have that

$$
\begin{aligned}
I I_{11} \leq & \int_{G_{m}^{2}}\left|\sum_{i=1}^{M_{k}-2} A_{p-M_{k}+i}^{-\alpha-2} \sum_{l=1}^{i} \bar{D}_{l}(u) \bar{D}_{l}(v)\right| d \mu(u, v) \\
& +\int_{G_{m}^{2}}\left|A_{p-1}^{-\alpha-1} \sum_{i=1}^{M_{k}-1} \bar{D}_{i}(u) \bar{D}_{i}(v)\right| d \mu(u, v) \\
\leq & c(\alpha)\left\{\sum_{v=1}^{M_{k}-2}\left(p-M_{k}+i\right)^{-\alpha-2} i+(p-1)^{-\alpha-1} M_{k}\right\} \\
\leq & c(\alpha)\left\{\sum_{i=1}^{\infty} i^{-\alpha-1}+M_{k}^{-\alpha}\right\}<\infty .
\end{aligned}
$$

The estimation of $I I_{12}$ and $I I_{13}$ are analogous to the estimation of $I I_{11}$. Applying Abel's transformation, in view of Lemma 1, we find that

$$
\begin{aligned}
I I_{12} \leq & \int_{G_{m}^{2}} D_{M_{k}}(u)\left|\sum_{i=1}^{M_{k}-2} A_{p-M_{k}+i}^{-\alpha-2} \sum_{l=1}^{i} \bar{D}_{l}(v)\right| d \mu(u, v) \\
& +\int_{G_{m}^{2}} D_{M_{k}}(u)\left|A_{p-1}^{-\alpha-1} \sum_{i=1}^{M_{k}-1} \bar{D}_{i}(v)\right| d \mu(u, v) \\
\leq & c(\alpha)\left\{\sum_{\nu=1}^{M_{k}-2}\left(p-M_{k}+i\right)^{-\alpha-2} i+(p-1)^{-\alpha-1} M_{k}\right\} \\
\leq & c(\alpha)\left\{\sum_{i=1}^{\infty} i^{-\alpha-1}+M_{k}^{-\alpha}\right\}<\infty
\end{aligned}
$$

and

$$
\begin{aligned}
I I I_{12} \leq & \int_{G_{m}^{2}} D_{M_{k}}(v)\left|\sum_{i=1}^{M_{k}-2} A_{p-M_{k}+i}^{-\alpha-2} \sum_{l=1}^{i} \bar{D}_{l}(u)\right| d \mu(u, v) \\
& +\int_{G_{m}^{2}} D_{M_{k}}(v)\left|A_{p-1}^{-\alpha-1} \sum_{i=1}^{M_{k}-1} \bar{D}_{i}(u)\right| d \mu(u, v)
\end{aligned}
$$




$$
\begin{aligned}
& \leq c(\alpha)\left\{\sum_{v=1}^{M_{k}-2}\left(p-M_{k}+i\right)^{-\alpha-2} i+(p-1)^{-\alpha-1} M_{k}\right\} \\
& \leq c(\alpha)\left\{\sum_{i=1}^{\infty} i^{-\alpha-1}+M_{k}^{-\alpha}\right\}<\infty .
\end{aligned}
$$

The proof is complete by combining (12)-(18).

Proof of Lemma 5 Let

$$
n=n_{k_{1}} M_{k_{1}}+\cdots+n_{k_{s}} M_{k_{s}}, \quad k_{1}>\cdots>k_{s} \geq 0 .
$$

Denote

$$
n^{(i)}=n_{k_{i}} M_{k_{i}}+\cdots+n_{k_{s}} M_{k_{s}}, \quad i=1,2, \ldots, s .
$$

Since (see [20])

$$
D_{j+n_{A} M_{A}}=D_{n_{A} M_{A}}+\psi_{n_{A} M_{A}} D_{j}
$$

we find that

$$
\begin{aligned}
I I I \leq & \int_{G_{m}^{2}}\left|\sum_{i=1}^{n_{k_{1}} M_{k_{1}}} A_{n-i}^{-\alpha-1} D_{i}(u) D_{i}(v)\right| d \mu(u, v) \\
& +\int_{G_{m}^{2}}\left|\sum_{i=1}^{n^{(2)}} A_{n^{(2)}-i}^{-\alpha-1} D_{i}(u) D_{i}(v)\right| d \mu(u, v) \\
& +\int_{G_{m}^{2}} D_{n_{k_{1}} M_{k_{1}}}(u) D_{n_{k_{1}} M_{k_{1}}}(v)\left|\sum_{i=1}^{n^{(2)}} A_{n^{(2)}-i}^{-\alpha-1}\right| d \mu(u, v) \\
& +\int_{G_{m}^{2}} D_{n_{k_{1}} M_{k_{1}}}(u)\left|\sum_{i=1}^{n^{(2)}} A_{n^{(2)}-i}^{-\alpha-1} D_{i}(v)\right| d \mu(u, v) \\
& +\int_{G_{m}^{2}} D_{n_{k_{1}} M_{k_{1}}}(v)\left|\sum_{i=1}^{n^{(2)}} A_{n^{(2)}-i}^{-\alpha-1} D_{i}(u)\right| d \mu(u, v) \\
:= & I I I_{1}+I I I_{2}+I I I_{3}+I I I_{4}+I I I_{5},
\end{aligned}
$$

where the first, second, third, fourth, and fifth terms on the right side of inequality (20) are denoted by $I I I_{1}, I I I_{2}, I I I_{3}, I I I_{4}$, and $I I I_{5}$, respectively.

By (1) we have that

$$
I I I_{3} \leq c(\alpha) .
$$

Moreover, since (see [24])

$$
\left|\sum_{i=1}^{n} A_{n-i}^{-\alpha-1} D_{i}(u)\right|=O\left(|u|^{\alpha-1}\right),
$$


for $\mathrm{III}_{4}$, we get that

$$
\begin{aligned}
I I I_{4} & \leq \int_{G_{m}^{2}} D_{n_{k_{1}} M_{k_{1}}}(u)|v|^{\alpha-1} d \mu(u, v) \\
& \leq \int_{G_{m}}|v|^{\alpha-1} d \mu(v)=\frac{1}{\alpha}<\infty .
\end{aligned}
$$

Analogously, we find that

$$
\begin{aligned}
I I I_{5} & \leq \int_{G_{m}^{2}} D_{n_{k_{1}} M_{k_{1}}}(v)|u|^{\alpha-1} d \mu(u, v) \\
& \leq \int_{G_{m}}|u|^{\alpha-1} d \mu(v)=\frac{1}{\alpha}<\infty .
\end{aligned}
$$

For $r \in\left\{0, \ldots m_{A}-1\right\}$ and $0 \leq j<M_{A}$ (see [20]), this yields that

$$
D_{j+r M_{A}}=\left(\sum_{q=0}^{r-1} \psi_{M_{A}}^{q}\right) D_{M_{A}}+\psi_{M_{A}}^{r} D_{j} .
$$

Thus we have

$$
\begin{aligned}
\int_{G_{m}^{2}} & \sum_{i=1}^{n_{k_{1}} M_{k_{1}}-1} A_{n-i}^{-\alpha-1} D_{i}(u) D_{i}(v) d \mu(u, v) \\
\leq & \int_{G_{m}^{2}} \sum_{r=0}^{n_{k_{1}}-1} \sum_{i=0}^{M_{k_{1}}-1} A_{n-i-r M_{k_{1}}}^{-\alpha-1} D_{i+r M_{k_{1}}}(u) D_{i+r M_{k_{1}}}(v) d \mu(u, v) \\
\leq & \int_{G_{m}^{2}} \sum_{r=0}^{n_{k_{1}}-1} \sum_{i=0}^{M_{k_{1}}-1} A_{n-i-r M_{k_{1}}}^{-\alpha-1}\left(\sum_{q=0}^{r-1} \psi_{M_{k_{1}}}^{q}\right) D_{M_{k_{1}}}(u) \\
& \times \sum_{q=0}^{r-1} \psi_{M_{k_{1}}}^{q} D_{M_{k_{1}}}(v) d \mu(u, v) \\
& +\int_{G_{m}^{2}} \sum_{r=0}^{n_{k_{1}}-1} \sum_{M_{k_{1}}-1} A_{n-i-r M_{k_{1}}}^{-\alpha-1}\left(\sum_{q=0}^{r-1} \psi_{M_{k_{1}}}^{q}\right) D_{M_{k_{1}}}(u) \psi_{M_{A}}^{r} D_{i}(v) d \mu(u, v) \\
& +\int_{G_{m}^{2}} \sum_{r=0}^{n_{k_{1}}-1} \sum_{i=0}^{M_{k_{1}}-1} A_{n-i-r M_{k_{1}}}^{-\alpha-1} \psi_{M_{A}}^{r} D_{i}(u)\left(\sum_{q=0}^{r-1} \psi_{M_{k_{1}}}^{q}\right) D_{M_{k_{1}}}(v) d \mu(u, v) \\
& +\int_{G_{m}^{2}} \sum_{r=0}^{n_{k_{1}}-1} \sum_{M_{k_{1}-1}-1} A_{n-i-r M_{k_{1}}}^{-\alpha-1} \psi_{M_{A}}^{r} D_{i}(u) \psi_{M_{A}}^{r} D_{i}(v) d \mu(u, v) .
\end{aligned}
$$

On the other hand, by (1) and (4) we obtain that

$$
\int_{G_{m}^{2}} A_{n-n_{k_{1}} M_{k_{1}}}^{-\alpha-1} D_{n_{k_{1}} M_{k_{1}}}(u) D_{n_{k_{1}} M_{k_{1}}}(v) d \mu(u, v) \leq c(\alpha) .
$$


Consequently, for $I I I_{1}$, we have the estimate

$$
\begin{aligned}
I I I_{1} \leq & \int_{G_{m}^{2}} D_{M_{k_{1}}}(u) D_{M_{k_{1}}}(v)\left|\sum_{r=0}^{n_{k_{1}}-1} \sum_{i=1}^{M_{k_{1}}} A_{n-i-r M_{k_{1}}}^{-\alpha-1}\right| d \mu(u, v) \\
& +\int_{G_{m}^{2}} D_{M_{k_{1}}}(u)\left|\sum_{r=0}^{n_{k_{1}}-1} \sum_{i=1}^{M_{k_{1}}} A_{n-i-r M_{k_{1}}}^{-\alpha-1} D_{i}(v)\right| d \mu(u, v) \\
& +\int_{G_{m}^{2}} D_{M_{k_{1}}}(v)\left|\sum_{r=0}^{n_{k_{1}}-1} \sum_{i=1}^{M_{k_{1}}} A_{n-i-r M_{k_{1}}}^{-\alpha-1} D_{i}(u)\right| d \mu(u, v) \\
& +\int_{G_{m}^{2}}\left|\sum_{r=0}^{n_{k_{1}}-1} \sum_{i=1}^{M_{k_{1}}} A_{n-i-r M_{k_{1}}}^{-\alpha-1} D_{i}(u) D_{i}(v)\right| d \mu(u, v)+c(\alpha) \\
:= & I I I_{11}+I I I_{12}+I I I_{13}+I I I_{14}+c(\alpha),
\end{aligned}
$$

where the first, second, third, and fourth terms on the right side of inequality (25) are denoted by $I I_{11}, I I_{12}, I I I_{13}$, and $I I_{14}$, respectively.

From Lemma 4 we have that

$$
I I I_{14} \leq c(\alpha) .
$$

The estimation of $I I I_{11}$ is analogous to that of $I I_{3}$, and we find that

$$
I I I_{11} \leq c(\alpha) .
$$

The estimation of $I I I_{12}$ and $I I I_{13}$ is analogous to that of $I I_{4}$, and we obtain that

$$
I I I_{12}<\infty
$$

and

$$
I I I_{13}<\infty
$$

After substituting (21) and (23)-(29) into (20), we conclude that

$$
\begin{aligned}
& \int_{G_{m}^{2}}\left|\sum_{i=1}^{n} A_{n-i}^{-\alpha-1} D_{i}(u) D_{i}(v)\right| d \mu(u, v) \\
& \leq \int_{G_{m}^{2}}\left|\sum_{i=1}^{n^{(2)}} A_{n^{(2)}-i}^{-\alpha-1} D_{i}(u) D_{i}(v)\right| d \mu(u, v)+c(\alpha) \\
& \leq \cdots \leq \int_{G_{m}^{2}}\left|\sum_{i=1}^{n^{(s)}} A_{n^{(s)}-i}^{-\alpha-1} D_{i}(u) D_{i}(v)\right| d \mu(u, v)+c(\alpha) s \\
& \quad \leq c(\alpha)+c(\alpha) s \leq c(\alpha) \log n .
\end{aligned}
$$

The proof is complete. 
Now we are ready to prove the main results.

Proof of Theorem 1 It is evident that

$$
\begin{aligned}
& \left\|\sigma_{M_{k}}^{-\alpha}(f)-f\right\|_{p} \\
& \leq \frac{1}{A_{M_{k}-1}^{-\alpha}}\left\|\int_{G_{m}^{2}} \sum_{i=1}^{M_{k-1}} A_{M_{k}-i}^{-\alpha-1} D_{i}(u) D_{i}(v)[f(\cdot-u, \cdot-v)-f(\cdot, \cdot)] d \mu(u, v)\right\|_{p} \\
& \quad+\frac{1}{A_{M_{k}-1}^{-\alpha}}\left\|\int_{G_{m}^{2}} \sum_{i=M_{k-1}+1}^{M_{k}} A_{M_{k}-i}^{-\alpha-1} D_{i}(u) D_{i}(v)[f(\cdot-u, \cdot-v)-f(\cdot, \cdot)] d \mu(u, v)\right\|_{p} \\
& :=I+I I .
\end{aligned}
$$

From Lemma 5 it follows that

$$
I \leq c(\alpha) \sum_{r=0}^{k-2} \frac{M_{r}}{M_{k}}\left(\omega_{1}\left(f, 1 / M_{r}\right)_{p}+\omega_{2}\left(f, 1 / M_{r}\right)_{p}\right) .
$$

Moreover, for $I I$, we have the estimate

$$
\begin{aligned}
I I \leq & \frac{1}{A_{M_{k}-1}^{-\alpha}} \| \int_{G_{m}^{2}} \sum_{i=M_{k-1}+1}^{M_{k}} A_{M_{k}-i}^{-\alpha-1} D_{i}(u) D_{i}(v) \\
& \times\left[f(\cdot-u, \cdot-v)-S_{M_{k-1}}^{(1)}(\cdot-u, \cdot-v, f)\right] d \mu(u, v) \|_{p} \\
& +\frac{1}{A_{M_{k}-1}^{-\alpha}} \| \int_{G_{m}^{2}} \sum_{i=M_{k-1}+1}^{M_{k}} A_{M_{k}-i}^{-\alpha-1} D_{i}(u) D_{i}(v) \\
& \times\left[S_{M_{k-1}}^{(1)}(\cdot-u, \cdot-v, f)-f(\cdot, \cdot)\right] d \mu(u, v) \|_{p} \\
:= & I I_{1}+I I_{2}
\end{aligned}
$$

where the first and second terms on the right side of inequality (32) are denoted by $I I_{1}$ and $\mathrm{II}_{2}$, respectively.

In view of the generalized Minkowski inequality, by (4) and Lemma 5 we get that

$$
\begin{aligned}
I I_{1} \leq & \frac{1}{A_{M_{k}-1}^{-\alpha}} \int_{G_{m}^{2}}\left|\sum_{i=M_{k-1}+1}^{M_{k}} A_{M_{k}-i}^{-\alpha-1} D_{i}(u) D_{i}(v)\right| \\
& \times\left\|f(\cdot-u, \cdot-v)-S_{M_{k-1}}^{(1)}(\cdot-u, \cdot-v, f)\right\|_{p} d \mu(u, v) \\
\leq & c(\alpha) M_{k}^{\alpha} \omega_{1}\left(f, 1 / M_{k-1}\right)_{p} .
\end{aligned}
$$

The estimation of $I I_{2}$ is analogous to that of $I I_{1}$, and we find that

$$
I I_{2} \leq c(\alpha) M_{k}^{\alpha} \omega_{2}\left(f, 1 / M_{k-1}\right)_{p}
$$

Combining (30)-(34), we obtain the proof of Theorem 1. 
Proof of Theorem 2 It is evident that

$$
\begin{aligned}
& \left\|\sigma_{n}^{-\alpha}(f)-f\right\|_{p} \\
& \leq \frac{1}{A_{n-1}^{-\alpha}}\left\|\int_{G_{m}^{2}} \sum_{i=1}^{M_{k-1}} A_{n-i}^{-\alpha-1} D_{i}(u) D_{i}(v)[f(\cdot-u, \cdot-v)-f(\cdot, \cdot)] d \mu(u, v)\right\|_{p} \\
& \quad+\frac{1}{A_{n-1}^{-\alpha}}\left\|\int_{G_{m}^{2}} \sum_{i=M_{k-1}+1}^{M_{k}} A_{n-i}^{-\alpha-1} D_{i}(u) D_{i}(v)[f(\cdot-u, \cdot-v)-f(\cdot, \cdot)] d \mu(u, v)\right\|_{p} \\
& \quad+\frac{1}{A_{n-1}^{-\alpha}}\left\|\int_{G_{m}^{2}} \sum_{i=M_{k}+1}^{n} A_{n-i}^{-\alpha-1} D_{i}(u) D_{i}(v)[f(\cdot-u, \cdot-v)-f(\cdot, \cdot)] d \mu(u, v)\right\|_{p} \\
& :=I+I I+I I I,
\end{aligned}
$$

where the first, second, and third terms on the right side of inequality (35) are denoted by $I, I I$, and $I I I$, respectively.

From Lemma 4 it follows that

$$
I \leq c(\alpha) \sum_{r=0}^{k-2} \frac{M_{r}}{M_{k}}\left(\omega_{1}\left(f, 1 / M_{r}\right)_{p}+\omega_{2}\left(f, 1 / M_{r}\right)_{p}\right) .
$$

Next, we repeat the arguments just in the same way as in the proof of Theorem 1 and find that

$$
I I \leq c(\alpha) M_{k}^{\alpha}\left(\omega_{1}\left(f, 1 / M_{k-1}\right)_{p}+\omega_{2}\left(f, 1 / M_{k-1}\right)_{p}\right) .
$$

On the other hand, for III, we have

$$
\begin{aligned}
I I I \leq & \frac{1}{A_{n-1}^{-\alpha}} \| \int_{G_{m}^{2}} \sum_{i=M_{k}+1}^{n} A_{n-i}^{-\alpha-1} D_{i}(u) D_{i}(v) \\
& \times[f(\cdot-u, \cdot-v)-f(\cdot, \cdot)] \|_{p} d \mu(u, v) \\
\leq & \frac{1}{A_{n}^{-\alpha}} \| \int_{G_{m}^{2}} \sum_{i=M_{k}+1}^{n} A_{n-i}^{-\alpha-1} D_{i}(u) D_{i}(v) \\
& \times\left[f(\cdot-u, \cdot-v)-S_{M_{k}, M_{k}}(\cdot-u, \cdot-v, f)\right] d \mu(u, v) \|_{p} \\
\leq & \frac{1}{A_{n}^{-\alpha}} \| \int_{G_{m}^{2}} \sum_{i=M_{k}+1}^{n} A_{n-i}^{-\alpha-1} D_{i}(u) D_{i}(v) \\
& \times\left[S_{M_{k}, M_{k}}(\cdot-u, \cdot-v, f)-S_{M_{k}, M_{k}}(\cdot, \cdot, f)\right] d \mu(u, v) \|_{p}
\end{aligned}
$$




$$
\begin{aligned}
& \leq \frac{1}{A_{n}^{-\alpha}} \| \int_{G_{m}^{2}} \sum_{i=M_{k}+1}^{n} A_{n-i}^{-\alpha-1} D_{i}(u) D_{i}(v) \\
& \quad \times\left[S_{M_{k}, M_{k}}(\cdot, \cdot, f)-f(\cdot, \cdot)\right] d \mu(u, v) \|_{p} \\
& :=I I I_{1}+I I I_{2}+I I I_{3},
\end{aligned}
$$

where the first, second, and third terms on the right side of inequality (38) are denoted by $I I I_{1}, I I I_{2}$, and $I I I_{3}$, respectively.

It is easy to show that

$$
I I I_{2}=0 .
$$

By the generalized Minkowski inequality and Lemma 5, for $I I I_{1}$, we obtain that

$$
\begin{aligned}
I I I_{1} \leq & \frac{1}{A_{n}^{-\alpha}} \int_{G_{m}^{2}}\left|\sum_{i=M_{k}+1}^{n} A_{n-i}^{-\alpha-1} D_{i}(u) D_{i}(v)\right| \\
& \times\left\|f(\cdot-u, \cdot-v)-S_{M_{r}, M_{r}}(\cdot-u, \cdot-v, f)\right\|_{p} d \mu(u, v) \\
\leq & c(\alpha) M_{k}^{\alpha}\left(\omega_{1}\left(f, 1 / M_{k-1}\right)_{p}+\omega_{2}\left(f, 1 / M_{k-1}\right)_{p}\right) \\
& \times \int_{G_{m}^{2}}\left|\sum_{v=M_{k}+1}^{n} A_{n-v}^{-\alpha-1} D_{v}(u) D_{v}(v)\right| d \mu(u, v) \\
\leq & c(\alpha) M_{k}^{\alpha} \log n\left(\omega_{1}\left(f, 1 / M_{k-1}\right)_{p}+\omega_{2}\left(f, 1 / M_{k-1}\right)_{p}\right) .
\end{aligned}
$$

The estimation of $I I I_{3}$ is analogous to that of $I I I_{2}$, and we find that

$$
I I I_{3} \leq c(\alpha) M_{k}^{\alpha} \log n\left(\omega_{1}\left(f, 1 / M_{k-1}\right)_{p}+\omega_{2}\left(f, 1 / M_{k-1}\right)_{p}\right) .
$$

After substituting (36)-(37) and (41) into (35), we obtain the proof of Theorem 2.

\section{Acknowledgements}

The authors would like to thank the referees for helpful suggestions.

\section{Funding}

Not applicable.

\section{Competing interests}

The authors declare that they have no competing interests.

\section{Authors' contributions}

The authors contributed equally to the writing of this paper. Both authors approved the final version of the manuscript.

\section{Publisher's Note}

Springer Nature remains neutral with regard to jurisdictional claims in published maps and institutional affiliations.

Received: 22 August 2018 Accepted: 2 December 2018 Published online: 19 December 2018

\section{References}

1. Agaev, G.N., Vilenkin, N.Ya., Dzhafarli, G.M., Rubinshtejn, A.I.: Multiplicative Systems of Functions and Harmonic Analysis on Zero-Dimensional Groups, Ehlm, Baku (1981) (in Russian)

2. Fine, N.J.: Cesàro summability of Walsh-Fourier series. Proc. Natl. Acad. Sci. USA 41, 558-591 (1995) 
3. Gát, G.: On the pointwise convergence of Cesàro means of two-variable functions with respect to unbounded Vilenkin systems. J. Approx. Theory 128(1), 69-99 (2004)

4. Gát, G.: Almost everywhere convergence of Fejér means of $L^{1}$ functions on rarely unbounded Vilenkin groups. Acta Math. Sin. Engl. Ser. 23(12), 2269-2294 (2007)

5. Gát, G.: On almost everywhere convergence of Fourier series on unbounded Vilenkin groups. Publ. Math. (Debr.) 75(1-2), 85-94 (2009)

6. Gát, G.: Some convergence and divergence results with respect to summation of Fourier series on one- and two-dimensional unbounded Vilenkin groups. Ann. Univ. Sci. Bp. Rolando Eötvös Nomin., Sect. Comput. 33, 157-173 (2010)

7. Gát, G., Blahota, I.: Norm summability of Nörlund logarithmic means on unbounded Vilenkin groups. Anal. Theory Appl. 24(1), 1-17 (2008)

8. Gát, G., Goginava, U.: Almost everywhere convergence of $(C, \alpha)$-means of quadratical partial sums of double Vilenkin-Fourier series. Georgian Math. J. 13(3), 447-462 (2006)

9. Gát, G., Goginava, U.: A weak type inequality for the maximal operator of $(C, \alpha)$-means of Fourier series with respect to the Walsh-Kaczmarz system. Acta Math. Hung. 125(1-2), 65-83 (2009)

10. Gát, G., Goginava, U.: Norm convergence of double Fourier series on unbounded Vilenkin groups. Acta Math. Hung 152(1), 201-216 (2017)

11. Gát, G., Goginava, U.: Norm convergence of logarithmic means on unbounded Vilenkin groups. Banach J. Math. Anal. 12(2), 422-438 (2018)

12. Glukhov, V.A.: On the summability of multiple Fourier series with respect to multiplicative systems. Mat. Zametki 39 665-673 (1986) In Russian

13. Goginava, U.: On the uniform convergence of Walsh-Fourier series. Acta Math. Hung. 93(1-2), 59-70 (2001)

14. Goginava, U.: On the approximation properties of Cesàro means of negative order of Walsh-Fourier series. J. Approx. Theory 115(1), 9-20 (2002)

15. Goginava, U.: Uniform convergence of Cesàro means of negative order of double Walsh-Fourier series. J. Approx. Theory 124(1), 96-108 (2003)

16. Goginava, U.: On Cesàro means of double trigonometric Fourier series. Mat. Zametki 74(4), 502-507 (2003) (Russian), translation in Math. Notes 74(3-4), 477-482 (2003)

17. Goginava, U.: Cesàro means of double Walsh-Fourier series. Anal. Math. 30(4), 289-304 (2004)

18. Goginava, U.: Approximation properties of $(C, \alpha)$ means of double Walsh-Fourier series. Anal. Theory Appl. 20(1), 77-98 (2004)

19. Goginava, U., Nagy, K.: On the maximal operator of Walsh-Kaczmarz-Fejér means. Czechoslov. Math. J. 61(3), 673-686 (2011)

20. Golubov, B.I., Efimov, A.V., Skvortsov, V.A.: Walsh Series and Transforms. Nauka, Moscow (1987) [in Russian]; English translation, Kluwer Academic, Dordrecht (1991)

21. Nagy, K.: Approximation by Cesàro means of negative order of Walsh-Kaczmarz-Fourier series. East J. Approx. 16(3), 297-311 (2010)

22. Schipp, F.: Über gewisse Maximaloperatoren. Ann. Univ. Sci. Bp. Rolando Eötvös Nomin., Sect. Math. 18, 189-195 (1975)

23. Schipp, F., Wade, W.R., Simon, P.: Walsh Series, an Introduction to Dyadic Harmonic Analysis. Hilger, Bristol (1990)

24. Shavardenidze, G.: On the convergence of Cesáro means of negative order of Vilenkin-Fourier series. arXiv:1811.08367

25. Simon, P., Weisz, F.: Weak inequalities for Cesàro and Riesz summability of Walsh-Fourier series. J. Approx. Theory $151(1), 1-19(2008)$

26. Tevzadze, V.I.: Uniform $(C, \alpha)(-1 \leq \alpha \leq 0)$ summability of Fourier series with respect to the Walsh-Paley system. Acta Math. Acad. Paedagog. Nyházi. 22(1), 41-61 (2006)

27. Zhizhiashvili, L.V.: Trigonometric Fourier Series and Their Conjugates, Revised and updated translation of "Some problems of the theory of trigonometric Fourier series and their conjugate series" (in Russian) [Tbilis. Gos. Univ. Tbilisi, 1993]; English translation: Kluwer Academic, Dordrecht (1996)

28. Zygmund, A.: Trigonometric Series, Vol. 1, Cambridge University Press, Cambridge (1959)

\section{Submit your manuscript to a SpringerOpen ${ }^{\circ}$ journal and benefit from:}

- Convenient online submission

- Rigorous peer review

- Open access: articles freely available online

- High visibility within the field

- Retaining the copyright to your article

Submit your next manuscript at $\gg$ springeropen.com 